\title{
Knowledge, Attitude and Practice of Breast Self-Examination amongst Women in Two Communities of Cameroon
}

\author{
Florent Ymele Fouelifack ${ }^{1,2,3}{ }^{*}$, Rene Pierre Binyom ${ }^{4}$, Anyimbi Mosman Ofeh ${ }^{1}$, \\ Jeanne Hortence Fouedjio ${ }^{2,5}$, Robinson Enow Mbu ${ }^{2,5}$
}

\begin{abstract}
${ }^{1}$ Department of Obstetrics and Gynecology of Higher Institute of Medical Technology of Nkolondom, Yaounde, Cameroon ${ }^{2}$ Obstetrics and Gynecology Unit of Yaounde Central Hospital, Yaounde, Cameroon

${ }^{3}$ Research, Education and Health Associative Group GARES-Falaise, Dschang, Cameroon

${ }^{4}$ Department of Surgery, Higher Institute of Medical Technology of Nkolondom, Yaounde, Cameroon

${ }^{5}$ Department of Obstetrics and Gynecology, Faculty of Medicine and Biomedical Sciences, University of Yaounde I, Yaounde, Cameroon Email: *yfouelifack@gmail.com
\end{abstract}

How to cite this paper: Fouelifack, F.Y., Binyom, R.P., Ofeh, A.M., Fouedjio, J.H. and Mbu, R.E. (2021) Knowledge, Attitude and Practice of Breast Self-Examination amongst Women in Two Communities of Cameroon. Open Journal of Obstetrics and Gynecology, 11, 773-793.

https://doi.org/10.4236/ojog.2021.116072

Received: May 21, 2021

Accepted: June 18, 2021

Published: June 21, 2021

Copyright $\odot 2021$ by author(s) and Scientific Research Publishing Inc. This work is licensed under the Creative Commons Attribution International License (CC BY 4.0).

http://creativecommons.org/licenses/by/4.0/

\begin{abstract}
Breast cancer is a major health problem in the world. It is the most common cancer in women and is equally the leading cause of cancer death in women. Though in controversy Breast self-examination (BSE) remains a useful tool in early diagnosis of breast cancer. The aim of this study was to evaluate the knowledge, attitude and practice of BSE amongst women in Yaoundé and Monatele. We carried out a cross sectional study in two communities of $\mathrm{Ca}$ meron over a period of eight months, targeting females older than 15 years. After obtaining authorization, we randomly interviewed 402 consenting participants, using a pretested questionnaire. Data were entered using CS Pro 7.3 and analysed using SPSS 23. Of a total of 402 participants, 256 (63.7\%) in Monatele and $146(36.3 \%)$ in Yaoundé VI, the mean age was $26.55 \pm 9.57$ years. Exactly $50 \%$ (201) had earlier heard of BSE. Health personnel and media represented the main sources of information on BSE. Of the total, 46 (11.4\%) knew BSE should be done monthly. Fifty-three percent had poor knowledge and $51.0 \%$ had a generally low attitude towards BSE. Out of 402 participants, $65.2 \%$ reported not practicing BSE. For those practicing, $71.6 \%$ had poor practices. The main reason for not practicing was lack of knowledge. Poor knowledge increased poor practice. In conclusion half of the women had heard about BSE even though the majority had a poor knowledge on BSE and very low overall attitude and practice. Health personnel and media played a key role in passing of information. Increasing the number of health campaigns, multiplying TV shows and health talks about breast selfexamination will improve knowledge and hence attitude and practice.
\end{abstract}




\section{Keywords}

Knowledge, Attitude, Practice, Breast, Self-Examination

\section{Introduction}

Breast cancer (BC) is a major health problem in the world. It is the most common cancer in women and is equally the leading cause of cancer death in women [1]. In 2018 BC was responsible for 2,088,849 of new cases of cancer (11.6\%) and 626,679 deaths (6.6\%). Approximately half of the breast cancer cases and $60 \%$ of the deaths are estimated to take place in developing countries. There is an increasing number of younger women being diagnosed with $\mathrm{BC}$ [2] [3].

In low-income countries and in sub-Saharan Africa, BC is responsible for one in five cancer deaths in women [4] [5]. Despite its emerging public health importance, incidence rates are still generally low in Africa, presumably below 35 per 100,000 women in most countries (as compared to over 90 - 120 per 100,000 women in most European or North American countries) [5]. In Cameroon, the incidence of BC is higher than the world's average; estimated at 2625 per 100,000 women with a resultant high mortality [6]. According to the Global Cancer Observatory (GLOBOCAN) 2012 estimation, BC is the most frequent cancer in women before the cervical cancer with an incidence rate of $31.5 \%$ (cited by sando $\mathrm{z}$ and co) and $35.1 \%$ in 2018 of all cancers in women [7] [8]. This can be explained by the existence of a breast and cervical cancer screening unit at the University Teaching Hospital of Yaoundé since 2009 and the organization of a cervical and breast cancer screening campaign in 2016. In addition, it is likely that doctors increased prescription of screening and histological examinations over this time period [9].

$\mathrm{BC}$ can manifest at late stages in several signs ranging from pain, skin changes, breast lump, abnormal breast discharges, nipple retraction and changes in breast size to subclinical forms where no mass can be palpated [10]. The screening methods for BC include breast self-examination (BSE), clinical breast examination and mammography, and these are usually done in combination [11]. BSE is the recommended method in developing countries like Cameroon because it is simple, private, costless, convenient and requires no specific equipment [1] [12].

In Africa, studies have been carried out to evaluate the Knowledge attitude and practice of BSE. In a study conducted among female undergraduates in University of Uyo, Akwa Ibom State in Nigeria, 23.4\% of respondents reported performing BSE regularly although $78.3 \%$ had heard of BSE [12]. In a similar study carried out among female undergraduate students in the University of Buea the main reason for not performing BSE as cited by the respondents was the lack of knowledge 73 (44\%) [1]. In Bambili in Cameroon, it was found out that as little as 7\% participants knew the appropriate time to perform a BSE [13]. 
Though still in controversy BSE is a screening technique for early BC detection that can be performed by women at home [6]. Evaluating women to show their knowledge, attitudes and practices concerning BSE is very important. That is why we carried out this study, with the objective to assess the knowledge, attitudes and practices (KAP) of BSE among women in our communities with respect to BC.

\section{Materials and Methods}

\subsection{Design, Duration, Period and Site of the Study}

It was a descriptive cross-sectional KAP type study, carried out over 6 months, from 15th January 2020 through 15th June 2020, in two communities: Yaoundé VI and Monatele in Cameroon.

Yaoundé VI is in the southwestern part of Yaoundé and is one of the largest in Yaoundé town. Monatele is the capital of the Lekie division in the Centre Region located about $70 \mathrm{~km}$ from Yaoundé. These communities were chosen because we had a good knowledge of them and we found it easy to interact with the inhabitants. They have a mixed population both English and French speaking, and several markets with usually many social gatherings. The houses in the neighbourhood are easily accessible making it relatively easy to interview women. They also have large populations attracted by the diverse economic activities of the area.

\subsection{Sampling and Procedure}

The study targeted women older than 15 years who could speak comprehensively or by means of a translator and who gave their consent. Were excluded all women who did not complete the interview or who did not completely fill the questionnaire. Sampling was done by cluster method at the level of the neighbourhoods and random sampling at a level of the households, markets, gatherings. Minimal sample size was estimated using the Lorenz formula [14]: $N=p^{*}$ $(1-p)^{*}(Z / d)^{2}$, where $N=$ sample size $p=0.47$ from research carried out among female undergraduate students in Cameroon [13], $Z=1.96, d=0.05$. This gave a minimal size of $N=383$ participants.

Our operational definition of BSE was a monthly examination of breasts conducted by women following the five major steps of BSE which include: examining both the breasts for size, shape, colour and contour while looking in front of the mirror with their arms straight, on the hips, and over the head, to palpate or feel the breast both in standing and lying position using the three finger pads [15]. For each participant, a questionnaire used had 4 sections: Socio-Demographic information and past history (21 questions), a second part which tested knowledge on BSE (11 questions), identification of attitude towards BSE (9 questions), Verification of BSE practice (8 questions). Knowledges, attitudes and practices levels were stratified according to poor, insufficient, satisfactory and good levels. 


\subsection{Data Analysis}

Data collected on questionnaires were entered into a data base using CS Pro version 7.3. Statistical analysis was carried out using Microsoft Excel 2007 and IBM SPSS version 23.0. The tools used to assess our results were the mean with its standard deviation, the median and the frequency. To seek associations between different variables, odds ratios with their $95 \%$ confidence intervals. $P$ was considered significant for any value less than $5 \%$.

\subsection{Ethical Considerations}

Ethical clearance $\mathrm{N}^{\circ} 2284$ IEC-UD/05/2020/T was obtained from the Ethical Committee of the University of Douala/Faculty of Medicine and Pharmaceutical Sciences. Administrative authorizations were also obtained from the Chief Medical Officer of the Centre Regional Ethics Committee for Research on Human Health Research and Monatele Health District. Each participant consented before being included, and all information obtained was treated with respect of confidentiality.

\section{Results}

A total of 509 women were approached (respectively 203 in Yaoundé IV and 306 in Monatele), 424 (respectively 155 and 269) gave their consent, from which 22 were excluded because of linguistic barriers. We finally recruited 402 women (146 in Yaoundé VI and 256 in Monatele) and analysed their interviews.

\subsection{Sociodemographic Characteristics}

For the 402 women, the mean age was $26.55 \pm 9.57$ years with extremes of 15 and 70 . The median age was 24 and the mode 20 years. The sociodemographic characteristics are presented in Table 1.

Table 1. Sociodemographic characteristics.

\begin{tabular}{cccc}
\hline Characteristics & response & Frequency $(\boldsymbol{n}=\mathbf{4 0 2})$ & $\%$ \\
\hline Age & $15-19$ & 89 & 22.1 \\
& $20-30$ & 218 & 54.2 \\
& $31-40$ & 62 & 15.4 \\
Community & $>40$ & 33 & 8.2 \\
Region of origin & Yaoundé VI & 146 & 36.3 \\
& Conatele & 256 & 63.7 \\
& Centre & 250 & 62.2 \\
& West & 60 & 14.9 \\
& Northwest & 55 & 13.7 \\
& East & 10 & 2.5 \\
& South & 8 & 2.0 \\
\hline
\end{tabular}




\begin{tabular}{|c|c|c|c|}
\hline \multicolumn{4}{|l|}{ Continued } \\
\hline & Southwest & 7 & 1.7 \\
\hline & others & 12 & 2.9 \\
\hline \multirow[t]{7}{*}{ Occupation } & Student & 165 & 41.0 \\
\hline & Housewife & 62 & 15.4 \\
\hline & Trader & 51 & 12.7 \\
\hline & Health personnel & 28 & 7.0 \\
\hline & Teacher & 18 & 4.5 \\
\hline & Other private sector & 72 & 17.9 \\
\hline & Other public sector & 6 & 1.5 \\
\hline \multirow[t]{2}{*}{ Marital status } & Unmarried & 250 & 62.2 \\
\hline & Married & 152 & 37.8 \\
\hline \multirow[t]{4}{*}{ Level of education } & Primary & 52 & 12.9 \\
\hline & Secondary/high school & 260 & 64.7 \\
\hline & University & 87 & 21.6 \\
\hline & No formal education & 3 & 0.7 \\
\hline \multirow[t]{3}{*}{ Religion } & Christian & 368 & 91.5 \\
\hline & Muslim & 18 & 4.5 \\
\hline & Others & 16 & 4.0 \\
\hline
\end{tabular}

The most represented age group was 20 - 30 (54.2\%), 256 (63.7\%) were interviewed at Monatele, 250 (62.2\%) were from the Centre Region origin, 165 (41\%) were students, 250 (62.2\%) were not married, 260 (64.7\%) had reached secondary school, and 368 (91.5\%) were Christians.

\subsection{Obstetrical and BC Past Histories of Participants}

The mean number of pregnancies was $2.18 \pm 2.3$ pregnancies with extremes of 0 and 10. The others obstetrical and breast cancer past histories of participants are presented in Table 2.

Of the 402 participants, 117 (29.1\%) had never been pregnant before, 190 (47.3\%) had 1 - 3 term deliveries, 390 (97\%) were of reproductive age, 301 (74.9\%) had no family history of BC, 96 (23.9\%) knew someone outside of their family with BC. Out of 269 who had given birth, 154 (57.2\%) did it before the age of 20 yrs.

\subsection{Knowledge}

Overall, 371 (92.3\%) of the participants had heard about BC meanwhile only $50 \%$ had heard of BSE. The following Table 3 summarizes the findings on Knowledge.

Overall, 371 (92.3\%) of the participants had heard about BC meanwhile only 50\% had heard of BSE. Health personnel represented the major source of information 
Table 2. Obstetrical and Breast cancer past histories of participants.

\begin{tabular}{|c|c|c|c|}
\hline Past history & response & Frequency $(n=402)$ & $\%$ \\
\hline \multirow[t]{3}{*}{ Number of pregnancies } & 0 & 117 & 29.1 \\
\hline & $1-3$ & 191 & 47.5 \\
\hline & $>3$ & 94 & 23.4 \\
\hline \multirow[t]{3}{*}{ Abortions } & 0 & 353 & 87.8 \\
\hline & $1-3$ & 46 & 11.4 \\
\hline & $>3$ & 3 & 0.7 \\
\hline \multirow[t]{3}{*}{ Live children } & 0 & 139 & 34.6 \\
\hline & $1-3$ & 195 & 48.5 \\
\hline & $>3$ & 68 & 16.9 \\
\hline \multirow[t]{3}{*}{${ }^{*}$ Age at first delivery } & $<20$ & 154 & 57.2 \\
\hline & $20-30$ & 113 & 42.0 \\
\hline & $>30$ & 2 & 0.7 \\
\hline \multirow[t]{2}{*}{ Reproductive period } & Yes & 390 & 97.0 \\
\hline & menopause & 12 & 3.0 \\
\hline \multirow[t]{3}{*}{ Menarche } & $<11$ & 3 & 0.7 \\
\hline & $11-15$ & 350 & 87.1 \\
\hline & $>15$ & 49 & 12.2 \\
\hline \multirow[t]{2}{*}{ Ever been diagnosed of $B C$} & Yes & 19 & 4.7 \\
\hline & No & 383 & 95.3 \\
\hline \multirow[t]{3}{*}{ Family history of BC } & Yes & 38 & 9.5 \\
\hline & No & 301 & 74.9 \\
\hline & Don't know & 63 & 15.7 \\
\hline \multirow[t]{2}{*}{ Knows someone outside of her family with BC } & Yes & 96 & 23.9 \\
\hline & No & 306 & 76.1 \\
\hline \multirow[t]{2}{*}{ Diagnosed of any other type of cancer } & Yes & 6 & 1.5 \\
\hline & No & 396 & 98.5 \\
\hline \multirow[t]{2}{*}{ Having a chronic condition } & Yes & 62 & 15.4 \\
\hline & no & 340 & 84.6 \\
\hline
\end{tabular}

*Not all women had given birth.

Table 3. Knowledge on BSE 1.

\begin{tabular}{cccc}
\hline variable & response & Frequency $(\boldsymbol{n}=\mathbf{4 0 2})$ & $\%$ \\
\hline Heard of BC & Yes & 371 & 92.3 \\
& No & 31 & 7.7 \\
Heard of BSE & Yes & 201 & 50.0 \\
& No & 201 & 50.0 \\
\hline
\end{tabular}




\section{Continued}

\begin{tabular}{|c|c|c|c|}
\hline \multirow[t]{8}{*}{${ }^{*}$ Sources of information on BSE } & Health personnel & 91 & 22.6 \\
\hline & Tv/radio & 51 & 12.7 \\
\hline & Teacher in school & 33 & 8.2 \\
\hline & friends & 32 & 8.0 \\
\hline & Newspaper & 25 & 6.2 \\
\hline & Health campaign & 18 & 4.5 \\
\hline & Internet & 16 & 4.0 \\
\hline & Family member & 8 & 2.0 \\
\hline \multirow[t]{3}{*}{ Frequent BSE can help detect BC } & Yes & 154 & 38.3 \\
\hline & No & 16 & 4.0 \\
\hline & Don't know & 232 & 57.7 \\
\hline \multirow[t]{3}{*}{ Early detection of $B C$ can improve survival } & Yes & 241 & 60 \\
\hline & No & 23 & 5.7 \\
\hline & Don’t know & 138 & 34.3 \\
\hline \multirow[t]{4}{*}{ Age at which BSE should begin } & Before 19 & 73 & 18.2 \\
\hline & $19-20$ & 10 & 2.5 \\
\hline & After 20 & 5 & 1.2 \\
\hline & Don't know, others & 314 & 78.1 \\
\hline \multirow[t]{6}{*}{ Frequency of BSE } & Monthly & 46 & 11.4 \\
\hline & Weekly & 26 & 6.5 \\
\hline & Daily & 47 & 11.7 \\
\hline & Every 3 months & 20 & 5.0 \\
\hline & Yearly & 14 & 3.5 \\
\hline & Don’t know & 249 & 61.9 \\
\hline \multirow[t]{5}{*}{ Period at which BSE should be done } & Before menses & 20 & 5.0 \\
\hline & $1-7$ days after menses & 42 & 10.4 \\
\hline & Anytime & 67 & 16.7 \\
\hline & During menses & 5 & 1.2 \\
\hline & Don't know & 268 & 66.7 \\
\hline \multirow[t]{5}{*}{ *What to look for when doing BSE } & Breast lump & 181 & 45 \\
\hline & Skin changes & 80 & 19.9 \\
\hline & Nipple discharge & 78 & 19.4 \\
\hline & Changes in shape/size & 113 & 28.1 \\
\hline & All four & 57 & 14.2 \\
\hline \multirow[t]{4}{*}{ *Positions in doing BSE } & Lying down & 94 & 23.4 \\
\hline & In front a mirror & 80 & 19.9 \\
\hline & Standing, showering & 98 & 24.4 \\
\hline & All three & 37 & 9.2 \\
\hline
\end{tabular}

*some participants had multiple sources of information and/or multiple responses. 
on BSE (22.6\%) followed by TV and radio (12.7\%); 154 (38.3\%) participants said BC can be detected by frequent BSE while 241 (60\%) of them said early detection of BC can improve survival chances. Only 10 (2.5\%) know the right age at which BSE should begin with 73 (18.2\%) saying BSE should begin before the age of 19 . Of the total, 46 (11.4\%) knew BSE should be done monthly and 42 (10.45) said BSE should be done 1 - 7 days after menses, 57 (14.2) knew all what to look for when doing BSE.

\section{Overall Knowledge on BSE}

Table 4 shows overall knowledge on BSE classified into four groups according to Essi et al. [16].

Out of 402 participants, 213 (53\%) had poor knowledge, 62 (15.4\%) had insufficient knowledge, 90 (22.4\%) had moderate while only 37 (9.2\%) had good knowledge.

\subsection{Attitude}

The following Table 5 summarizes the findings on attitude.

Table 4. Overall knowledge.

\begin{tabular}{ccc}
\hline Grade & Frequency $(\boldsymbol{n}=\mathbf{4 0 2})$ & $\%$ \\
\hline Poor & 213 & 53 \\
Insufficient & 62 & 15.4 \\
Moderate & 90 & 22.4 \\
good & 37 & 9.2 \\
\hline
\end{tabular}

Table 5. Attitude towards BSE.

\begin{tabular}{cccc}
\hline Variable & response & Frequency $(\boldsymbol{n}=\mathbf{4 0 2})$ & $\%$ \\
\hline Can find BC myself (doing BSE) & Yes & 121 & 30.1 \\
& No & 39 & 9.7 \\
& Maybe & 39 & 9.7 \\
Fear detecting BC & Don't know & 203 & 50.5 \\
& Yes & 113 & 28.1 \\
& No & 80 & 19.9 \\
BSE is useful/important & Maybe & 1 & 0.2 \\
& Don't know & 208 & 51.7 \\
& Yes & 190 & 47.3 \\
& No & 6 & 1.5 \\
BSE is disgraceful & Don't know & 196 & 48.8 \\
& Yes & 15 & 3.7 \\
& No & 182 & 45.3 \\
& Don't know & 205 & 50.9 \\
\hline
\end{tabular}




\section{Continued}

\begin{tabular}{cccc}
\hline $\begin{array}{c}\text { Health campaigns, tv shows etc } \\
\text { motivate me to do BSE }\end{array}$ & Yes & 179 & 44.5 \\
& No & 19 & 4.7 \\
Advice other women to d BSE & Yes & 204 & 50.7 \\
Need to learn more on BSE & Yes & 201 & 50 \\
BSE is a waste of time & no & 397 & 98.8 \\
& Yes & 5 & 1.2 \\
Overall attitude & No & 8 & 2.0 \\
& Don't know & 192 & 47.8 \\
& Low & 202 & 50.2 \\
& Moderate & 31 & 51.0 \\
\end{tabular}

Out of 402 participants, 121 (30.1\%) said they could find BC by themselves by doing BSE, 113 (28.1\%) feared detecting BC and 190 (47.3\%) said BSE is useful. Also, $182(45.3 \%)$ said BSE is not a disgraceful practice. Finally, 397 (98.8\%) of the participants were willing to learn about BSE. Overall, out of 402 participants, 205 (51.0\%) had a low attitude towards BSE.

\subsection{Practice}

Summary of practices is shown in Table 6.

Out of 420 participants, 262 (65.2\%) reported not practicing BSE while 140 (34.8\%) practiced it.

Of those who practiced BSE, 24 (17.2\%) did so between 1 and 7 days after their menses, 31 (22.1\%) reported starting BSE by the age of $19-20$ years, 37 (26.4\%) practiced BSE in all 3 positions while $39(27.8 \%)$ rightly used the palm and at least 3 fingers to palpate the breast, 47 (33.5\%) did not follow any particular pattern while doing BSE.

In summary the findings for the overall practice shows that 288 (71.6\%) participants had poor practice while only $5(1.2 \%)$ had good practice of BSE.

\section{Reasons for Not Practicing BSE}

Out of 262 participants who reported not practicing BSE, several reasons were mentioned: 172 (65.64\%) said they had not heard about it while 43 (16.41\%) said they didn't know how to do it.

\subsection{Associations between Variables}

To seek associations between variables, we used variables which had heterogeneous distribution. After dichotomization, we did binary logistic regressions to find independent associations between variables. 
Table 6. practice of BSE.

\begin{tabular}{|c|c|c|c|}
\hline variable & Response & $\begin{array}{c}\text { Frequency } \\
(n=\text { yes }=140)\end{array}$ & $\%$ \\
\hline \multirow[t]{2}{*}{ Practice BSE } & Yes & 140 & 34.8 \\
\hline & no & 262 & 65.2 \\
\hline \multirow{4}{*}{$\begin{array}{l}\text { Period during which I do BSE } \\
\quad \text { (if Practice BSE = yes) }\end{array}$} & Before menses & 11 & 7.9 \\
\hline & $1-7$ days after menses & 24 & 17.2 \\
\hline & Anytime & 103 & 73.6 \\
\hline & During menses & 2 & 1.4 \\
\hline \multirow[t]{4}{*}{ Age at start of BSE } & Before 19 & 43 & 30.7 \\
\hline & $19-20$ & 31 & 22.1 \\
\hline & After 20 & 52 & 37.1 \\
\hline & forgotten & 14 & 10.0 \\
\hline \multirow[t]{5}{*}{${ }^{*}$ Positions in which I do BSE } & Standing, showering & 88 & 62.8 \\
\hline & Lying down & 64 & 45.7 \\
\hline & In front mirror & 47 & 33.6 \\
\hline & others & 20 & 5.0 \\
\hline & All three positions & 37 & 18.4 \\
\hline \multirow[t]{4}{*}{ Part of hand used in BSE } & Palm only & 19 & 13.6 \\
\hline & Fingers only & 73 & 52.1 \\
\hline & Palm + at least 3 fingers & 39 & 27.8 \\
\hline & Palm and 2 fingers & 9 & 6.4 \\
\hline \multirow[t]{4}{*}{ Pattern of palpation } & Circular & 71 & 50.7 \\
\hline & Vertical & 10 & 7.1 \\
\hline & Quadrant by quadrant & 12 & 8.6 \\
\hline & No specific pattern & 47 & 33.5 \\
\hline \multirow[t]{4}{*}{ Overall practice } & Poor & 288 & 71.6 \\
\hline & Insufficient & 80 & 19.9 \\
\hline & Moderate & 29 & 7.2 \\
\hline & Good & 5 & 1.2 \\
\hline
\end{tabular}

*some participants had multiple positions.

\subsubsection{Associations between Sociodemographic Characteristics,} Obstetrical and BC Past Histories of Participants and Knowledge Associations between sociodemographic characteristics, obstetrical and BC past histories of participants and knowledge are shown in Table 7.

There was no independent association between sociodemographic, obstetrical characteristics, past history of $\mathrm{BC}$ and participant's knowledge.

\subsubsection{Association between Sociodemographic Characteristics,} Obstetrical and BC Past Histories of Participants and Attitude Association between sociodemographic characteristics, obstetrical and $\mathrm{BC}$ past histories of participants and attitude are shown in Table 8. 
Table 7. Associations between sociodemographic characteristics, obstetrical and BC past histories of participants and knowledge.

\begin{tabular}{|c|c|c|c|c|c|c|}
\hline \multirow{2}{*}{ Variable } & \multicolumn{2}{|c|}{ Knowledge on BSE $\mathrm{n}(\%)$} & \multirow{2}{*}{ OR (CI 95\%) } & \multirow{2}{*}{$\mathbf{P}$} & \multirow{2}{*}{ aOR (CI 95\%) } & \multirow{2}{*}{$\mathrm{aP}$} \\
\hline & Poor/insufficient & Moderate/good & & & & \\
\hline \multicolumn{7}{|l|}{ Age } \\
\hline$<30$ & $197(68.6)$ & $90(31.4)$ & $1.04(0.65-1.65)$ & 0.48 & & \\
\hline$\geq 30$ & $78(67.8)$ & $37(32.2)$ & & & & \\
\hline \multicolumn{7}{|l|}{ Level of education } \\
\hline University & $35(40.2)$ & $52(59.8)$ & $0.2(0.13-0.35)$ & $\leq 0.001$ & $0.51(0.25-1.03)$ & 0.06 \\
\hline Secondary/primary & $240(76.2)$ & $75(23.8)$ & & & & \\
\hline \multicolumn{7}{|l|}{ Marital status } \\
\hline Single & $173(69.2)$ & $77(30.8)$ & $1.1(0.72-1.69)$ & 0.37 & & \\
\hline Married/cohabitation & $102(67.1)$ & $50(32.9)$ & & & & \\
\hline \multicolumn{7}{|l|}{ Occupation } \\
\hline Student/teacher/health personnel & $128(60.7)$ & $83(39.3)$ & $0.46(0.3-0.7)$ & $\leq 0.001$ & $0.72(0.38-1.36)$ & 0.31 \\
\hline Trader/housewife/others & $147(77.0)$ & $44(23.0)$ & & & & \\
\hline \multicolumn{7}{|l|}{ Number of pregnancies } \\
\hline$<3$ & $183(68.5)$ & $84(31.5)$ & $1.01(0.65-1.59)$ & 0.51 & & \\
\hline$\geq 3$ & $92(68.1)$ & $43(31.9)$ & & & & \\
\hline \multicolumn{7}{|l|}{ Number of term deliveries } \\
\hline$<3$ & $188(66.0)$ & $97(34.0)$ & $0.67(0.41-1.08)$ & 0.06 & & \\
\hline$\geq 3$ & $87(74.4)$ & $30(25.6)$ & & & & \\
\hline \multicolumn{7}{|l|}{ Menarche } \\
\hline$\leq 15$ & $239(67.7)$ & $114(32.3)$ & $0.76(0.39-1.48)$ & 0.26 & & \\
\hline$>15$ & $36(73.5)$ & $13(26.5)$ & & & & \\
\hline \multicolumn{7}{|c|}{ Family history of $\mathrm{BC} / \mathrm{knows}$ someone with $\mathrm{BC}$} \\
\hline Yes & $77(61.1)$ & $49(38.9)$ & $0.62(0.39-0.97)$ & 0.02 & $1.00(0.54-1.85)$ & 0.98 \\
\hline No & $198(71.7)$ & $78(28.3)$ & & & & \\
\hline \multicolumn{7}{|l|}{ Community } \\
\hline Yaoundé VI & $101(69.2)$ & $45(30.8)$ & $1.06(0.68-1.64)$ & 0.45 & & \\
\hline Monatele & $174(68.0)$ & $82(32.0)$ & & & & \\
\hline
\end{tabular}

Table 8. Sample characteristics vs attitude.

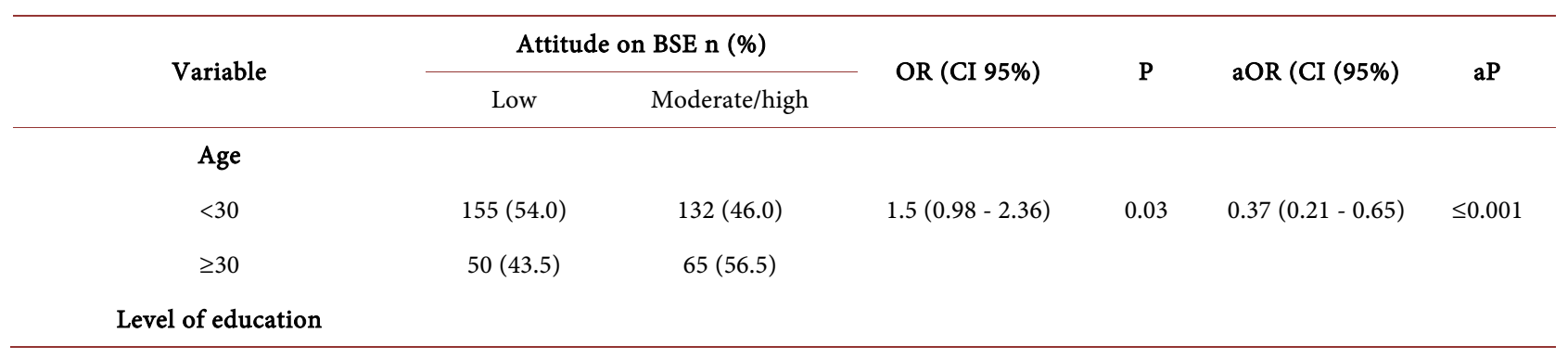




\section{Continued}

\begin{tabular}{|c|c|c|c|c|c|c|}
\hline University & $18(20.7)$ & $69(79.3)$ & $0.17(0.10-0.31)$ & $\leq 0.001$ & $0.17(0.09-0.33)$ & $\leq 0.001$ \\
\hline Secondary/primary & $187(59.4)$ & $128(40.6)$ & & & & \\
\hline \multicolumn{7}{|l|}{ Marital status } \\
\hline Single & $126(50.4)$ & $124(49.6)$ & $0.94(0.63-1.41)$ & 0.42 & & \\
\hline Married/cohabitation & $79(52.0)$ & $73(48.0)$ & & & & \\
\hline \multicolumn{7}{|l|}{ Occupation } \\
\hline Student/teacher/health personnel & $93(44.1)$ & $118(55.9)$ & $0.56(0.37-0.83)$ & $\leq 0.001$ & $0.53(0.32-0.90)$ & 0.01 \\
\hline Trader/housewife/others & $112(58.6)$ & $79(41.4)$ & & & & \\
\hline \multicolumn{7}{|l|}{ Number of pregnancies } \\
\hline$<3$ & $138(51.7)$ & $129(48.3)$ & $1.09(0.72-1.64)$ & 0.38 & & \\
\hline$\geq 3$ & $67(49.6)$ & $68(50.4)$ & & & & \\
\hline \multicolumn{7}{|l|}{ Number of term deliveries } \\
\hline$<3$ & $140(49.1)$ & $145(50.9)$ & $0.77(0.50-1.19)$ & 0.14 & & \\
\hline$\geq 3$ & $65(55.6)$ & $52(44.4)$ & & & & \\
\hline \multicolumn{7}{|l|}{ Menarche } \\
\hline$\leq 15$ & $184(52.1)$ & $169(47.9)$ & $1.45(0.79-2.65)$ & 0.14 & & \\
\hline$>15$ & $21(42.1)$ & $28(57.1)$ & & & & \\
\hline \multicolumn{7}{|c|}{ Family history of BC/knows someone with BC } \\
\hline Yes & $50(39.7)$ & $76(60.3)$ & $0.51(0.33-0.79)$ & 0.002 & $0.50(0.31-0.81)$ & 0.005 \\
\hline No & $155(56.2)$ & $121(43.8)$ & & & & \\
\hline \multicolumn{7}{|l|}{ Community } \\
\hline Yaoundé VI & $53(36.3)$ & $93(63.7)$ & $0.39(0.26-0.59)$ & $\leq 0.001$ & $0.37(0.21-0.65)$ & $\leq 0.001$ \\
\hline Monatele & $152(59.4)$ & $104(40.6)$ & & & & \\
\hline
\end{tabular}

Age ( $<30$ years), level of education (university), occupation (Student/teacher/ health personnel), family history of $\mathrm{BC} /$ knowing someone with breast cancer and being from Yaoundé community protected from poor attitude.

\subsubsection{Association between Sociodemographic Characteristics,} Obstetrical and BC Past Histories of Participants and Practice Association between sociodemographic characteristics, obstetrical and BC past histories of participants and practice are shown in Table 9.

Only a family history of breast cancer or knowing someone with breast cancer protected from poor/insufficient practice.

\subsubsection{Association between Knowledge, Attitudes and Practices, Obstetrical Characteristics, History of BC and Attitude of Participants}

Association between knowledge, attitudes and practices, obstetrical characteristics, history of breast cancer and attitude of participants are shown in Table 10.

Only Poor/insufficient knowledge independently increases Poor/insufficient 
Table 9. Sample characteristics vs Practice.

\begin{tabular}{|c|c|c|c|c|c|c|}
\hline \multirow{2}{*}{ Variable } & \multicolumn{2}{|c|}{ Practice of BSE $n(\%)$} & \multirow{2}{*}{ OR (CI 95\%) } & \multirow{2}{*}{$\mathbf{P}$} & \multirow{2}{*}{ aOR (CI 95\%) } & \multirow{2}{*}{ aP } \\
\hline & Poor/insufficient & Moderate/good & & & & \\
\hline \multicolumn{7}{|l|}{ Age } \\
\hline$<30$ & $263(91.6)$ & $24(8.4)$ & $1.04(0.48-2.25)$ & 0.53 & & \\
\hline$\geq 30$ & $105(91.3)$ & $10(8.7)$ & & & & \\
\hline \multicolumn{7}{|l|}{ Level of education } \\
\hline University & $71(81.6)$ & $16(18.4)$ & $0.27(1.3-0.55)$ & $\leq 0.001$ & $0.68(0.28-1.64)$ & 0.39 \\
\hline Secondary/primary & $297(94.3)$ & $18(5.7)$ & & & & \\
\hline \multicolumn{7}{|l|}{ Marital status } \\
\hline Single & $230(92.0)$ & $20(8.0)$ & $1.17(0.57-2.38)$ & 0.40 & & \\
\hline Married/cohabitation & $138(90.8)$ & $14(9.2)$ & & & & \\
\hline \multicolumn{7}{|l|}{ Occupation } \\
\hline Student/teacher/health personnel & $188(89.1)$ & $23(10.9)$ & $0.5(0.237-1.05)$ & 0.04 & $0.77(0.30-1.98)$ & 0.60 \\
\hline Trader/housewife/others & $180(94.2)$ & $11(5.8)$ & & & & \\
\hline \multicolumn{7}{|l|}{ Number of pregnancies } \\
\hline$<3$ & $244(91.4)$ & $23(8.6)$ & $0.94(0.44-1.99)$ & 0.51 & & \\
\hline$\geq 3$ & $124(91.9)$ & $11(8.1)$ & & & & \\
\hline \multicolumn{7}{|l|}{ Number of term deliveries } \\
\hline$<3$ & $260(91.2)$ & $25(8.8)$ & $0.87(0.39-1.92)$ & 0.44 & & \\
\hline$\geq 3$ & $108(92.3)$ & $9(7.7)$ & & & & \\
\hline \multicolumn{7}{|l|}{ Menarche } \\
\hline$\leq 15$ & $322(91.2)$ & $31(8.8)$ & $0.68(0.199-2.3)$ & 0.38 & & \\
\hline$>15$ & $46(93.9)$ & $3(6.1)$ & & & & \\
\hline \multicolumn{7}{|c|}{ Family history of $\mathrm{BC} /$ knows someone with $\mathrm{BC}$} \\
\hline Yes & $107(84.9)$ & $19(15.1)$ & $0.32(0.16-0.66)$ & 0.002 & $0.391(0.17-0.88)$ & 0.02 \\
\hline No & $261(94.6)$ & $15(5.4)$ & & & & \\
\hline \multicolumn{7}{|l|}{ Community } \\
\hline Yaoundé VI & $128(87.7)$ & $18(12.3)$ & $0.47(0.23-0.96)$ & 0.029 & $1.58(1.01-2.42)$ & 0.49 \\
\hline Monatele & $240(93.8)$ & $16(6.3)$ & & & & \\
\hline
\end{tabular}

Table 10. Association between knowledge, attitudes and practices, sources of information from health personnel and number of sources of information on BSE.

\begin{tabular}{|c|c|c|c|c|c|c|}
\hline variable & Poor/insufficient & Moderate/good & OR (CI 95\%) & $\mathbf{P}$ & aOR (CI 95\%) & aP \\
\hline \multicolumn{7}{|l|}{ Practice of BSE n (\%) } \\
\hline \multicolumn{7}{|l|}{ Knowledge } \\
\hline Poor/insufficient & $271(98.5)$ & $4(1.5)$ & $20.95(7.19-61.0)$ & $\leq 0.001$ & $6.25(1.94-20.13)$ & 0.002 \\
\hline Moderate/good & $97(76.4)$ & $30(23.6)$ & & & & \\
\hline Attitude & & & & & & \\
\hline
\end{tabular}




\section{Continued}

\begin{tabular}{|c|c|c|c|c|c|c|}
\hline Low & $201(100)$ & 0 & na & na & na & \\
\hline Moderate/high & $163(82.7)$ & $34(17.3)$ & & & & \\
\hline \multicolumn{7}{|l|}{ Knowledge on BSE } \\
\hline \multicolumn{7}{|c|}{ BSE from health personnel } \\
\hline No & $243(78.1)$ & $68(21.9)$ & $6.59(3.97-10.94)$ & $\leq 0.001$ & $1.09(0.59-1.99)$ & 0.78 \\
\hline Yes & $32(35.2)$ & $59(64.8)$ & & & & \\
\hline \multicolumn{7}{|l|}{ Number of sources } \\
\hline$<3$ & $75(40.3)$ & $111(59.7)$ & $9.46(1.22-73.47)$ & 0.006 & $0.13(0.01-1.07)$ & 0.05 \\
\hline$\geq 3$ & $1(6.7)$ & $14(93.3)$ & & & & \\
\hline \multicolumn{7}{|l|}{ Practice of BSE } \\
\hline \multicolumn{7}{|c|}{ BSE from health personnel } \\
\hline no & $295(94.9)$ & $16(5.1)$ & $4.55(2.21-9.34)$ & $\leq 0.001$ & $1.37(0.59-3.15)$ & 0.45 \\
\hline Yes & $73(80.2)$ & $18(19.8)$ & & & & \\
\hline \multicolumn{7}{|c|}{ Number of sources of info on BSE } \\
\hline$<3$ & $158(84.9)$ & $28(15.1)$ & $3.76(1.24-11.39)$ & 0.024 & $0.46(0.13-1.62)$ & 0.22 \\
\hline$\geq 3$ & $9(60.0)$ & $6(40.0)$ & & & & \\
\hline
\end{tabular}

practices of BSE. There was no independent association observed between others variables.

\section{Discussion}

\subsection{Sociodemographic Characteristics and Past History}

The mean age of the participants was $26.55 \pm 9.57$ years with extremes of 15 and 70. The age group of 20 - 30 was the most represented with 218 (54.2\%) participants out of 402 (Table 1). This result is similar to the findings in Buea in 2012 [17] and Nigeria in 2016 [18] but different from the findings in Douala; 30 - 39 in 2017 [19]. The later study was carried out among health professionals who in majority were older than the participants of our study group. Out of 402, 260 (64.7\%) had reached secondary school only, 250 (62.2\%) were from Centre Region, explained by the fact that this study was carried out in the Centre region (Table 1). Students made up 156 (41\%) of the participants different from the result of studies in Buea [1] and Bambili [13] where all the participants were students, 250 participants (62.2\%) were single and 368 (91.5\%) were Christians (Table 1), similar to the results in Ghana [20]. Cameroon [21] and Ghana are made up mostly of Christians [22].

Out of 402 participants, 117 (29.15\%) had never been pregnant before while 190 (47.3\%) had 1 - 3 term deliveries (Table 2), 390 (97\%) were of reproductive age close to findings in vietnam where $90.8 \%$ were of reproductive age [23]: the participants in both studies were less than 50 years old. Only 38 (9.5\%) had a family history of BC (Table 2). 


\subsection{Knowledge on BSE}

Table 3 shows the variables used to calculate the overall knowledge in Table 4. Half of the participants had heard about BSE before (Table 3). This was close to the findings in Bambili in 2017 where 47\% of the participants had heard about BSE [13]. This however is different from the findings in Buea in 2015 [1]; 73.5\% amongst university students, Buea in 2012 on BSE and BC awareness; $74.17 \%$ [17], Nigeria in 2017 among Female undergraduates in the University of Uyo [12]; 78.3\% and the United Arab Emirates in 2019; 68.9\% [24]. In our study less than half of the participants were students while other studies were carried out on students only.

A majority of the participants (92.3\%) had heard of BC (Table 3), close to 88.1\%, 88.8\% and 100\% found respectively in Bambili in 2017 [13], Malaysia in 2012 [25] and Nigeria [12]. Almost 38.3\% said BC can be detected early by frequent BSE (Table 3). This is lower than 51.7\% found in Nigeria [12], 88.6\% in Buea Cameroon [1] and $77.6 \%$ in Malaysia [25]. Six out of ten participants agreed that early detection of breast cancer can improve survival chances (Table 3). This is different from findings in Nigeria in 2017; 89.1\% [12], and Ethiopia in 2017 by Kalayu et al; 89.9\% [26].

Only $18.2 \%$ believed BSE should begin before 19 years while only $10(2.5 \%)$ knew the right age at which BSE should begin (Table 3), close to $3.2 \%$ found in Bambili in 2017 [2]. This opinion might be due to the fact that there is an increasing number in BC cases. Only $11.4 \%$ knew BSE should be done monthly. This percentage is lower than $37.3 \%, 25.9 \%, 62.9 \%$ and $58.4 \%$ found respectively in Buea in 2015 [1], Bambili in 2017 [13], Palestine in 2015 [15] and UAE in 2019 [24]. This lower level may be explained by the relatively low exposure to BSE information. A majority (61.9\%) did not have any idea of how often BSE should be done. This value almost triples that found in Nigeria in 2017 [12].

Health personnel (22.6\%) and media (TV/radio) (12.7\%) were the major sources of information on BSE in our study. In a study in Buea in 2015 health personnel $(15.1 \%)$ and peers $(12.7 \%)$ were the main factors that influenced performing BSE [1]. In 2015 in Palestine, 45.4\% were taught on it by health staff [15]. In Ethiopia in 2017 , the main sources of information were mass media (39.8\%) and health personnel $(22.3 \%)$ [26]. This shows that the two means of information are the most used.

Only $10.45 \%$ knew the right time BSE should be done (1 - 7 days after menses) (Table 3), close to 8.8\% and 7\%, found in Egypt [27] and in Bambili [13], but lower than $30.5 \%$ and $42.8 \%$ found in Ethiopia [26] and in the UAE [26]. About $66.7 \%$ participants did not know the right period to do BSE, $14.2 \%$ knew all what to look for when doing BSE and 9.2\% said BSE should be done in all three positions (Table 3). Similar results were found in Buea [1].

Overall, the level of good knowledge was 9.2\% (Table 4), close to $9.6 \%$ found in Buea [1], lower than $15.5 \%$ in Palestine [15] and 30.25\% in Ethiopia [26]. Of the total, $22.4 \%$ had moderate knowledge, and $68.4 \%$ had insufficient/poor know- 
ledge (Table 4). This poor knowledge is almost twice that found in Ethiopia (37.6\%) [26], and greater than $43.3 \%$ found in Palestine [15]. This difference may be explained by the fact that the later studies were carried out in a milieu where majority of the persons were university students.

\subsection{Attitude}

About $30.1 \%$ of participants said they could find BC by themselves by doing BSE (Table 5). This value was greater than $8.7 \%$ found in the UAE in 2019 [24], and lower than 59\% found in Buea in 2015 [1]. Only 28.1\% feared detecting BC (Table 5), value close to $26.5 \%$ and $29.9 \%$ found in Buea [1] and Palestine [15]. These close levels may be explained by the fact that $\mathrm{BC}$ is perceived as a terminal disease and is the leading cause of cancer death in women [1].

In our study, $44.5 \%$ were motivated by campaigns and TV shows (Table 5 ). This result is lower than $81.9 \%$ in Buea [1]. About $47.3 \%$ of the participants found BSE is useful (Table 5). This finding is lower than 93\%, 96.3\%, 88.6\% found in Bambili in 2017 [13], Ghana in 2018 [20], and Buea in 2015. This observed difference may be due to the difference in overall knowledge on BSE. BSE was considered a waste of time for only $2 \%$ of the participants (Table 5 ). This is lower than $6.3 \%$ found in 2018 in Ghana [20]. In the later study more participants had heard of and practiced BSE.

Finally, $98.8 \%$ of the participants were willing to learn or learn more about BSE (Table 5), 51.0\% had a generally low attitude towards BSE, different from $2.4 \%$ found in Buea in 2015 [1]. This may be explained by the fact that participants in the later study had better knowledge and perception of the importance of BSE.

\subsection{Practice}

Only $65.2 \%$ reported not practicing BSE (Table 6). This result is close to $72.3 \%$ and $71.7 \%$ found in the UAE in 2019 [24] and Ethiopia in 2017 [26]. Out of 140 (34.8\%) who practiced BSE, 24 (17.2\%) did so between 1 and 7 days after their menses (Table 6). In the South West Region of Cameroon in 2018, 93.8\% of women who practiced BSE did not wait to perform it at a specific time of the month, whereas $3.1 \%$ performed BSE the week and $0.3 \%$ the second week following their menses [28]. In their studies $74.6 \%$ had not heard of BSE [28] while $50 \%$ of our participants had heard of BSE before (Table 5).

Out of 140 who had practiced BSE, $77.6 \%$ did so within the past month (Table 6). In a study in Buea in 2015, 29.5\% claimed to have performed BSE within the past 12 months [1]. Major reasons for not practicing BSE were lack of knowledge on BSE (65.64\%) and not knowing how to (16.41\%). Similar reasons were found in 2015 in Buea [1], Ethiopia in 2017 [26] and the United Arab Emirates [24]. Lack of knowledge plays an important role in BSE practice.

Overall, $71.6 \%$ of the participants had poor practice, $19.9 \%$ insufficient practice while only a critically low $8.4 \%$ had moderate to good practice. This result was different from that found in the United Arab Emirates in 2015: 15.5\% of 
good practice [15] [19] and in Douala in 2017 where practice was estimated as very poor in $28.3 \%$, poor in $36.4 \%$, good in $9.7 \%$, and excellent in $25.6 \%$. This difference can be explained by the fact that the later studies were carried out among nursing students and health professionals respectively who have a relatively higher knowledge of breast cancer and BSE (Table 4).

\subsection{Associations between Variables}

In our stuy, there was no independent association between sociodemographic, obstetrical characteristics, past history of $\mathrm{BC}$ and participant's knowledge (Table 7). Age ( $<30$ years) $(\mathrm{aOR}=0.373(0.214-0.650)$; $\mathrm{p}$ : 0.001$)$, level of education (university) (aOR: $0.179(0.097-0.332) \mathrm{p}<0.001)$, occupation (Student/teacher/ health personnel) (aOR: $0.538(0.320-0.904) \mathrm{p}: 0.019)$ and a family history of $\mathrm{BC} /$ knowing someone with $\mathrm{BC}$ (aOR: $0.507(0.316-0.813) \mathrm{p}$ : 0.005$)$ and being from Yaoundé community (aOR: $0.373(0.214-0.650) \mathrm{p}$ : 0.001$)$ protected from poor attitude (Table 8). Only a family history of BC or knowing someone with BC protected from Poor/insufficient practice (aOR: $0.391(0.173-0.882)$ p: 0.024) (Table 9). Only Poor/insufficient knowledge independently increases Poor/insufficient practices of BSE (aOR: 6.251 (1.941 - 20.133) P: 0.002) (Table 10). Same result was found in Buea [1].

\section{Study Limitations and Confounding Factors}

Even though this study was carried out in two communities, it does not give an overall view of the knowledge attitude and practices of BSE of women in the Centre region or women in other subdivisions of Yaoundé. The responses of the women on BSE practices may not reflect what they actually do as the women were not watched practicing BSE. This was a confounding factor.

\section{Conclusion}

BSE remains a mean of early detection of BC in Cameroon. In our study, the majority of participants had poor overall knowledge on BSE and very low overall attitude and good practice. Health personnel and media play a very important role in passing of information. Women having a family history of breast cancer or who know someone with breast cancer tend to have better attitude and practice of BSE. Lack of knowledge is the main reason for not practicing BSE. Intersectoral and interdisciplinary interventions to intensify the provider behavior change communication, to ameliorate knowledge, attitude and practices about BSE and BC should be increased in our communities. Although many results here obtained overlapped those previously reported in Africa, we believe that area-specific data is important.

\section{Authors' Contribution}

Florent Y Fouelifack and Anyimbi M Ofeh conceived the study, wrote-up, and edited the manuscript. Rene P Binyom and Jeanne H Fouedjio wrote-up and 
edited the manuscript. Robinson E Mbu supervised the study from its conception up to write-up of the manuscript. All the authors read and approved the final manuscript.

\section{Data Availability}

The data used to support the findings of this study are available from the corresponding author upon request.

\section{Funding Statement}

Apart from the personal contribution of the authors, we have not received any assistance or any promises of any kind, to date for this work. This is valid for all authors. Aside the individual contribution of each of the authors, this study received no financial assistance.

\section{Acknowledgements}

The authors heartily appreciate the administrative authorities of Yaoundé and Monatele, for having eased in carrying as well as community leaders who announced in churches, parks, markets and other social gatherings that they were going to received researchers. We equally thank all participants who enabled us to obtain all necessary information toward the publishing of this study.

\section{Disclosures and Ethics}

Before carrying out the study, we obtained Ethical Clearance $\mathrm{N}^{\circ} 2284$ IEC-UD/ 05/2020/T of Institutional Ethics Committee for Research on Human Health of University of Douala (IEC-UDo), signed on the 19th of May 2020, and the Ethical Clearance $\mathrm{N}^{\circ} 3345 / \mathrm{CRERSHC} / 2020$ of the Centre Regional Ethics Committee for Research on Human Health Research, signed on the 4th of March 2020. We also obtained research authorisations $\mathrm{N}^{\circ}$-3402/AP/MINSANTE/SG/DRSPC/ CRERSH signed on 06th of March 2020 and $\mathrm{N}^{\circ}$-CDS96/L/MNSP/DRSPC/DSM signed on 05th of March 2020 by the Chief Medical Officer of Monatele Health District. After meeting each woman, we wished to recruit, we presented ourselves to her. We then explained the aim of our study and the aim of data collection and the possible outcomes. Also, the information collected was kept confidential. We taught each woman who wanted to know (know more) about BSE. All information obtained or used in the study was treated as confidential by questionnaire coding and used for the study only.

\section{Conflicts of Interest}

The authors declare that there is no conflict of interest regarding the publication of this paper.

\section{References}

[1] Nde, F.P., Assob, J.C.N., Kwenti, T.E., Njunda, A.L. and Tainebe, T.R.G. (2015) 
Knowledge, Attitude and Practice of Breast Self-Examination among Female Undergraduate Students in the University of Buea Women's Health. BMC Research Notes, 8, 4-9. https://doi.org/10.1186/s13104-015-1004-4

[2] Assi, H.A., Khoury, K.E., Dbouk, H., Khalil, L.E., Mouhieddine, T.H. and El Saghir, N.S. (2013) Epidemiology and Prognosis of Breast Cancer in Young Women. Journal of Thoracic Disease, 5, S2-S8.

[3] Bray, F., Ferlay, J., Soerjomataram, I., Siegel, R.L., Torre, L.A. and Jemal, A. (2018) Global Cancer Statistics 2018: GLOBOCAN Estimates of Incidence and Mortality Worldwide for 36 Cancers in 185 Countries. CA: A Cancer Journal for Clinicians, 68, 394-424. https://doi.org/10.3322/caac.21492

[4] Dibisa, T.M., Gelano, T.F., Negesa, L, Haraweya, T.G. and Abate, D. (2019) Breast Cancer Screening Practice and Its Associated Factors among Women in Kersa District, Eastern Ethiopia. The Pan African Medical Journal, 33, 1-10. https://doi.org/10.11604/pamj.2019.33.144.18062

[5] Brinton, L.A., Figueroa, J.D., Awuah, B., Yarney, J, Wiafe, S., Wood, S.N., et al. (2014) Breast Cancer in Sub-Saharan Africa: Opportunities for Prevention. Breast Cancer Research and Treatment, 144, 467-478. https://doi.org/10.1007/s10549-014-2868-Z

[6] Bray, F., Ren, J.-S., Masuyer, E. and Ferlay, J. (2013) Global Estimates of Cancer Prevalence for 27 Sites in the Adult Population in 2008. International Journal of Cancer, 132, 1133-1145. https://doi.org/10.1002/ijc.27711

[7] Sando, Z., Fouogue, J.T., Fouelifack, F.Y., Fouedjio, J.H., Mboudou, E.T. and Essame, J.L.O. (2014) Profil des cancers gynécologiques et mammaires à Yaounde-Cameroun. The Pan African Medical Journal, 17, 28. https://doi.org/10.11604/pamj.2014.17.28.3447

[8] Salem, A.A.S., Salem, M.A.E. and Abbass, H. (2010) Breast Cancer: Surgery at the South Egypt Cancer Institute. Cancers, 2, 1771-1778. https://doi.org/10.3390/cancers2041771

[9] Kemo, A.D., Nkegoum, B., Nangue, C., Djuikwo, F., Bita'a, L.B., Tebeu, P.M., et al. (2019) Histo-Epidemiological Aspects of Gynecological and Breast Cancers at the University Teaching Hospital of Yaoundé. The Pan African Medical Journal, 33, 130. https://doi.org/10.11604/pamj.2019.33.130.18874

[10] American Cancer Society. Breast Cancer Signs and Symptoms. Most Common Symptoms.

https://www.cancer.org/cancer/breast-cancer/about/breast-cancer-signs-and-sympt oms.html

[11] Leung, J., McKenzie, S., Martin, J., Dobson, A. and McLaughlin, D. (2014) Longitudinal Patterns of Breast Cancer Screening: Mammography, Clinical, and Breast Self-Examinations in a Rural and Urban Setting. Women's Heal Issues, 24, 139-146. https://doi.org/10.1016/j.whi.2013.11.005

[12] Akpanekpo, E. (2017) Knowledge, Attitude and Practice of Breast Self-Examination (BSE) among Female Undergraduates in the University of Uyo, Southern Nigeria. EVO Journal of Public Health, 2, 6-11.

[13] Sama, C.B., Dzekem, B., Kehbila, J., Ekabe, C.J., Vofo, B. and Abua, N.L. (2017) Awareness of Breast Cancer and Breast Self-Examination among Female Undergraduate Students in a Higher Teacher Training College in Cameroon. The Pan African Medical Journal, 28, 91. https://doi.org/10.11604/pamj.2017.28.91.10986

[14] Charan, J. and Biswas, T. (2013) How to Calculate Sample Size for Different Study Designs in Medical Research? Indian Journal of Psychological Medicine, 35, 121- 
126. https://doi.org/10.4103/0253-7176.116232

[15] Ayed, A., Faeda, E., Lubna, H., Imad, F., Sew, N., Talahmeh, B., et al. (2015) Breast Self-Examination in Terms of Knowledge, Attitude, and Practice among Nursing Students of Arab American University/Jenin. Journal of Education and Practice, 6, 27-31.

[16] Essi, M.J. and Njoya, O. (2013) L'Enquête CAP (Connaissances, Attitudes, Pratiques) en Recherche Médicale. Health Sciences and Disease, 14, 1-3.

[17] Suh, M., Atashili, J., Fuh, E.A. and Eta, V.A. (2012) Breast Self-Examination and Breast Cancer Awareness in Women in Developing Countries: A Survey of Women in Buea, Cameroon. BMC Research Notes, 5, 11. https://doi.org/10.1186/1756-0500-5-627

[18] Nwaneri, A., Osuala, E.O., Okpala, P.U., Emesowum, A.C. and Iheanacho, P. (2017) Knowledge and Awareness of Breast Cancer among Rural Women in Umuowa Orlu Local Government Area Imo State, South East, Nigeria. Nigerian Journal of Clinical Practice, 20, 489-494.

[19] Nguefack, C.T., N'djeudjui, C., Engbang, J.P.N., Nana, T.N., Ekane, G.H. and Tebeu, P.M. (2018) Knowledge, Attitude, and Practice on Breast Cancer among Health Professionals in Douala References Hospitals, Cameroon. Journal of Cancer Education, 33, 457-462. https://doi.org/10.1007/s13187-016-1158-3

[20] Fondjo, L.A., Owusu-Afriyie, O., Sakyi, S.A., Wiafe, A.A., Amankwaa, B. and Acheampong, E. (2018) Comparative Assessment of Knowledge, Attitudes, and Practice of Breast Self-Examination among Female Secondary and Tertiary School Students in Ghana. International Journal of Breast Cancer, 2018, Article ID: 7502047. https://doi.org/10.1155/2018/7502047

[21] Cameroon-United States Department of State. https://www.state.gov/reports/2019-report-on-international-religious-freedom/cam eroon

[22] Ghana-United States Department of State. https://www.state.gov/reports/2019-report-on-international-religious-freedom/gha $\underline{\text { na }}$

[23] Tuyen, D.Q., Dung, T.V., Dong, V.H., Kien, T.T., Huon, T., et al. (2019) Breast SelfExamination: Knowledge and Practice among Female Textile Workers in Vietnam. Cancer Control, 26, 1-7. https://doi.org/10.1177/1073274819862788

[24] Rahman, S.A., Al-Marzouki, A., Otim, M., Khayat, N.E.H.K., Yousuf, R. and Rahman, P. (2019) Awareness about Breast Cancer and Breast Self-Examination among Female Students at the University of Sharjah: A Cross-Sectional Study. Asian Pacific Journal of Cancer Prevention, 20, 1901-1908. https://doi.org/10.31557/APJCP.2019.20.6.1901

[25] Al-Naggar, R.A., Bobryshev, Y.V. and Al-Jashamy, K. (2012) Practice of Breast SelfExamination among Women in Malaysia. Asian Pacific Journal of Cancer Prevention, 13, 3829-3833. https://doi.org/10.7314/APJCP.2012.13.8.3829

[26] Birhane, K., Alemayehu, M., Anawte, B., Gebremariyam, G., Danial, R., Addis, S., et al. (2017) Practices of Breast Self-Examination and Associated Factors among Female Debre Berhan University Students. International Journal of Breast Cancer, 2017, Article ID: 8026297. https://doi.org/10.1155/2017/8026297

[27] Boulos, D.N.K. and Ghali, R.R. (2014) Awareness of Breast Cancer among Female Students at Ain Shams University, Egypt. Global Journal of Health Science, 6, 154 161. https://doi.org/10.5539/gjhs.v6n1p154

[28] Azemfac, K., Christie, S.A., Carvalho, M.M., Nana, T., Fonje, A.N., Halle-Ekana, H., 
et al. (2019) A Community-Based Assessment of Knowledge and Practice of Breast Self-Examination and Prevalence of Breast Disease in Southwest Cameroon. Journal of Cancer Epidemiology, 2019, Article ID: 2928901.

https://doi.org/10.1155/2019/2928901 